\title{
Statistical and spectral texture analysis methods for discrimination of bread crumb images
}

\author{
Gonzales-Barron, U. A.* and Butler, F. \\ Biosystems Engineering, School of Agriculture, Food Science and Veterinary Medicine, University \\ College Dublin, Dublin 2, Ireland \\ *Corresponding author: ursula.gonzalesbarron@ucd.ie
}

\begin{abstract}
The objectives of this study were: (i) to assess the performance of three statistical texture analysis methods (grey-level co-occurrence matrix, neighbourhood grey-level difference matrix and geometrical method) and two spectral texture analysis methods (Fourier power spe ctrum features and spectral features sensitive to additive noise) to differentiate bread crumb grain loaves produced with five different flours, and (ii) to find the textural features that presented high discriminant capacity. The stepwise-selected textural features from the neighbourhood difference matrix and the geometrical method could classify $85 \%$ and $80 \%$ of the samples by flour type, respectively, using cross-validation. As a whole, the statistical texture analysis depicted the bread crumb visual texture better than the transform methods as the factor analysis for the two principal components for the statistical methods explained 91.2 $-95.9 \%$ of the total variability. The method of wedges and rings from the Fourier power spectrum performed poorly for distinguishing the bread crumb grains produced with the different flours (correct classification of $58 \%$ and total variability accounted for of $75.4 \%$ ). Stepwise discriminant analysis carried out over all textural features selected eleven features with the highest discriminant capacity and factor analysis identified the presence of three major components in which the bread crumb grain appearance normally varied: grain fineness, grain homogeneity and grain isotropy. This model accounted for $93 \%$ of the total variability and, thus, is preferable for discriminating bread crumb visual appearance.
\end{abstract}

Keywords: bread crumb, grain, appearance, visual texture, image analysis, texture analysis

\section{Introduction}

The texture of an image or the texture of a segmented area is an important feature for area description, which quantifies some characteristic of the grey level variation within the object. Generally speaking, textures are complex visual patterns composed of entities, or subpatterns that give rise to the perceived lightness, uniformity, density, roughness, regularity, linearity, frequency, phase, directionality, coarseness, randomness, fineness, smoothness, granulation, etc. (Levine, 1985). Many methods are available to characterise the textural appearance of materials directly from grey level images. Statistical methods are used extensively in texture classification - identifying the given textured region from a given set of textured classes. Textures that are random in nature are well suited for statistical characterization (Jain, 1989). Spectral methods are based on the Fourier transform to study the global periodicity of an image. Among the texture analysis methods for food quality evaluation, most approaches are statistical, including the co-occurrence matrix method (McCauley et al., 1994; Park and Chen, 1996; Shiranita et al., 1998; Basset et al., 2000) and the pixel-value run length method (Li et al., 1999; Majumdar and Jayas, 2000; Basset et al., 2000). However, several texture description methods are based on Fourier spectrum and wavelet transforms (Li et al., 2001; Whittaker et al., 1992). 
In the case of bread crumb texture classification, three texture analysis methods have been used to discriminate among types of bread loaves. Textural features from first-order statistical measures and co-occurrence matrices were used in multivariate discriminant analysis to differentiate between two bread types (Zayas, 1993). They gave 97\% correct classification, while the use of image textural features extracted from the Haar transform (Bertrand et al., 1993) gave $82 \%$ correct classification of seven types of bread loaves produced with different surfactants.

The objectives of this study were: (1) to assess the performance of three statistical texture analysis methods (grey-level co-occurrence matrix, neighbourhood grey-level difference matrix, and geometrical method), and two spectral texture analysis methods (Fourier power spectrum features and spectral features insensitive to additive noise) to differentiate bread crumb grain of loaves produced with different flours, and (2) to ascertain the underlying factors in which bread crumb visual appearance normally vary.

\section{Materials and Methods}

The bread used was from a study (Keehan et al., 2003) investigating the baking characteristics of four organic flours and a control flour. The organic flours were provided by Doves Farm 'Biobake strong white bakers flour' (Irish Agent, Wholefoods Wholesale Ltd., Dublin, Ireland), Rank Hovis, 'organic white bakers flours' (Rank Hovis, The Millers (RHM), Dublin, Ireland), Allied Mills, 'strong organic white flour' (Irish Agent, Daly Food Ingredients, Cork, Ireland), and Shiptons Mills, 'traditional organic white four (strong No. 4)' (Shiptons Mills Ltd., Gloucestershire, U.K.). An Irish milled non-organic strong white flour was used as a control (Odlum Group, Dublin, Ireland). Later in this work, the flours will be addressed as Doves, Rank, Allied, Shiptons and Odlum.

Five loaves per flour type, taken from two baking batches, were sliced using an electric slicer to obtain 15-mm thick slices. The slices were placed on a copy stand against a dark background, illuminated by two $100 \mathrm{~W}$ incandescent lamps (angle of illumination was $65^{\circ}$ ) placed at opposite sides of the sample $25 \mathrm{~cm}$ from the slice plane. Images of seven central slices from each loaf were acquired without compression with a spatial resolution of 1 pixel line $\approx 0.0635 \mathrm{~mm}$ using a DC260 Kodak (KODAK Co., NY) digital camera, positioned at 30 $\mathrm{cm}$ from the sample. The colour images were transferred by the KODAK Access TWAIN Acquire software (DC220/DC260 Software, KODAK Co., NY) to a Dell PC (1GHz $128 \mathrm{MB})$ without any image compression and saved as TIFF format. Using Image Tool v.2.0 software (UTHSCSA, University of Texas Health Science Centre, San Antonio, Texas), the centre of each image (slice) was cropped to a square of 630 x 630 pixels (representing $40 \times 40 \mathrm{~mm}^{2}$ of the slice area) and converted to grey-level image ( 8 bits). For a given treatment, 35 images ( 5 loaves $\mathrm{x} 7$ slices) were acquired. However, to eliminate outliers, images that presented very large pores or holes were not considered for the multivariate statistical evaluation. A total of 171 bread crumb images were analysed. To discriminate among bread crumb images produced with different flours, three approaches of statistical texture analysis and two transform or spectral methods were utilised. 


\section{$\underline{\text { Grey-level co-occurrence matrices (GLCOM) }}$}

This method, developed by Haralick et al. (1973), computes a series of matrices that describe the spatial variation of grey level values. A variety of features that describe their overall characteristics can be computed on these matrices. GLCO is the 2-dimensional distribution based on the number of occurrences of going from grey level $i$ to grey level $j$, given that the intersample distance is $d$ and the direction is the angle $\theta$. The distance $d$ was 1 pixel line and the angles $\theta$ were $0^{\circ}, 45^{\circ}, 90^{\circ}$ and $135^{\circ}$. For each matrix, the angular second moment $\left(F_{1}\right.$ or $A S M)$, contrast $\left(F_{2}\right)$, correlation $\left(F_{3}\right)$, variance $\left(F_{4}\right)$, inverse difference moment $\left(F_{5}\right)$, entropy $\left(F_{9}\right)$, and an information measure of correlation $\left(F_{12}\right)$ were calculated, according to the equations given in Haralick et al. (1973). The textural features were averaged.

\section{Neighbourhood grey-level difference matrix (NGLDM)}

This statistical method was suggested by Amadasun and King (1989) in an attempt to define texture measures correlated with human perception of textures. The entries of a NGLD matrix are computed based on measuring the difference between the intensity level of a pixel and the average intensity computed over a square sliding window centred at the pixel. The neighborhood size for this window was 3 pixels. Five features were derived from the NGLDM, to quantitatively describe perceptual texture properties: coarseness $\left(f_{\text {cos }}\right)$, contrast $\left.f_{\text {con }}\right)$, busyness $\left(f_{\text {bus }}\right)$, complexity $\left(f_{\text {com }}\right)$, and textures strength $\left(f_{\text {str }}\right)$, according to the equations given in Amadasun and King (1989).

\section{$\underline{\text { Statistical geometrical features }}$}

This method (Chen et al., 1995) consists of producing a stack of binary images from a grey level image and calculating features from the connected objects of the binary images. When an image $f(x, y)$ is thresholded with a threshold value $t, 1 \leq t \leq G-1$ (G represents grey levels), a corresponding binary image is obtained $f(x, y ; t)$. So for a given original image, there are $G-1$ potentially different binary images. This set of images is termed a binary image stack. Let the number of connected regions of 1 -valued pixels in the binary image $f(x, y ; t)$ be denoted by $N O C(t)$. NOC $(t)$ is a function of $t, 1 \leq t \leq G-1$. To each of the connected regions, a measure of irregularity $(I R G L)$ or un-compactness is applied. The two functions of $t$, i.e., $N O C(t)$ and $I R G L(t)$ are characterised using the following four statistics: maximum value, average value, sample mean and standard deviation of the sample. In total, eight features were extracted.

\section{Textural features insensitive to additive noise}

Liu and Jernigan (1990) proposed texture measures extracted in the spatial frequency domain that are insensitive to additive noise. Measures of the shape of the spatial frequency spectrum such as location, size and orientation of peaks and entropy of the normalised spectrum in regions of spatial frequency can be used to discriminate textures. According to the procedure given in Liu and Jernigan (1990), the following features were calculated: energy in major peak $\left(f_{1}\right)$, Laplacian of major peak $\left(f_{2}\right)$, Laplacian of secondary peak $\left(f_{3}\right)$, relative orientation of major and secondary peaks $\left(f_{6}\right)$, isotropy of power spectrum $\left(f_{7}\right)$, squared distance between major and secondary peak $\left(f_{13}\right)$, principal component magnitude square $\left(f_{14}\right)$, ratio of minor to major axis $\left(f_{16}\right)$, moment of inertia of quadrant I $\left(f_{17}\right)$, moment of inertia of quadrant II $\left(f_{18}\right)$, moment ratio $\left(f_{19}\right)$, percentage energy in quadrant I $\left(f_{20}\right)$, percentage energy of quadrant II $\left(f_{21}\right)$, Laplacian of major peak phase $\left(f_{23}\right)$, Laplacian of secondary peak phase $\left(f_{24}\right)$ and relative entropy of power spectrum $\left(f_{25}\right)$. 


\section{$\underline{\text { Rings and wedges from Fourier power spectrum }}$}

It is well known that the radial distribution of values in the Fourier power spectrum $P(u, v)$ is sensitive to texture coarseness (Weszka et al. 1976). A coarse texture will have high values of $P(u, v)$ concentrated near the origin, while in a fine texture, the values of $P(u, v)$ will be more spread out. Thus, a set of features that should be useful are the averages of $P(u, v)$ taken over ring-shaped regions $(R)$ centred at the origin, ie, features of the form

$$
R_{1-2}=\sum_{r_{1}}^{r_{2}} P(u, v), \quad r_{1}^{2} \leq u^{2}+v^{2}<r_{2}^{2}, 0 \leq u, v \leq n-1
$$

for various values of $r$, the ring radius.

Similarly, the angular distribution of values in $P(u, v)$ is sensitive to the directionality of the texture in $f(x, y)$ (Weszka et al. 1976). If a texture is orientated in a given direction $\theta$, it will have high values of $P(u, v)$ concentrated around the perpendicular direction $\theta+(\pi / 2)$, while in a non-directional texture, $P(u, v)$ should also be nondirectional. Thus, a set of energy features for analysing texture directionality are taken over wedge-shaped regions $(W)$ centred at the origin by using,

$$
W_{1-2}=\sum_{\theta_{1}}^{\theta_{2}} P(u, v), \quad \theta_{1} \leq \tan ^{-1}(v / u)<\theta_{2}, 0 \leq u, v \leq n-1
$$

for various values of $\theta$, the wedge slope. The ring shaped regions were computed for the radii $\left[r_{1}, r_{2}\right]=[2,4>,[4,8>,[8,16>,[16,32>$, and $[32,64>$. The wedge-shaped regions were computed for the angles $\left[\theta_{1}, \theta_{2}\right]=\left[-22.5^{\circ}, 22.5^{\circ}>,\left[22.5^{\circ}, 67.5^{\circ}>,\left[67.5^{\circ}, 112.5^{\circ}>\right.\right.\right.$, and $\left[112.5^{\circ}, 157.5^{\circ}>\right.$. Also all possible intersections of rings and wedges were computed.

All the textural methods were programmed in Matlab 6.12 (The MathWorks Inc., Natick, Mass, USA).

\section{$\underline{\text { Statistical Analysis }}$}

The 171 bread crumb images produced five matrices containing textural features (one matrix for each texture method). Matrices of $171 \times 7,171 \times 5,171 \times 8,171 \times 15$, and $171 \times 29$ corresponded to the results of the co-occurrence matrix method, neighbourhood grey-level difference matrix method, geometrical method, spectral features insensitive to additive noise, and wedges and rings from the Fourier power spectrum, respectively. A stepwise discriminant analysis (SDA) was carried out on each matrix to identify the flour types from the textural features. SDA makes it possible to introduce variables one after the other in the prediction while taking their discrimination ability into account. Thus, for each method, the number of textural features was reduced to keep the ones with the highest discriminant power $(\mathrm{sls}=\mathrm{sle}=0.15)$. Since, the chi-squared test of equality of covariance matrices were not significant at the 0.1 level for any texture method, quadratic discrimination analysis was performed in preference to linear discrimination analysis (Hair et al., 1998). The classification performance on flour type was evaluated by cross-validation (Der and Everitt, 2002).

For each texture analysis method, rotated factor analysis using the varimax rotation by the principal components technique was performed for the textural features selected by the 
stepwise discriminant analysis. In all cases, the eigenvalue or screeplot (Hair et al., 1998) led to a solution of two factors. Finally, in order to find out and summarise the pattern of intercorrelations among textural features, factor analysis was performed on the textural variables selected by the stepwise discriminant analysis applied to a matrix containing all the textural features (64 in total). Here, a solution of three principal components was considered. The statistical analysis was carried out in SAS version 8.2 (SAS Institute Inc, NC, USA).

\section{Results and Discussion}

\section{Grey-level co-occurrence matrix method}

The stepwise discriminant analysis applied to the features from the co-occurrence matrices selected the angular second moment $\left(\mathrm{F}_{1}\right)$, contrast $\left(\mathrm{F}_{2}\right)$, variance $\left(\mathrm{F}_{4}\right)$, inverse difference moment $\left(\mathrm{F}_{5}\right)$, entropy $\left(\mathrm{F}_{9}\right)$ and correlation $\left(\mathrm{F}_{12}\right)$ as the variables with the highest predictive performance. The use of these variables for classification by cross-validation gave an average of $78.9 \%$ of correct identification (range $67-94 \%$ ). In Table 1, the rows give the actual bread treatments and the columns the treatments that were identified by SDA. For example, Allied was represented by 34 image samples, 23 samples were correctly identified, six samples were wrongly classified into Doves and five samples were wrongly classified into Shiptons. The numbers on the diagonal line of the table therefore correspond to correctly identified samples. Thus, the control treatment Odlum was highly differentiated $(94.1 \%$ correctly assigned samples) from the rest.

Table 1. Classification performance to segregate images by flour type using the method of the grey-level co-occurrence matrices

\begin{tabular}{lccccc}
\hline \multirow{2}{*}{$\begin{array}{l}\text { Flour } \\
\text { type }\end{array}$} & \multicolumn{5}{c}{ Classification with Cross-Validation } \\
\cline { 2 - 6 } Allied & 23 & Doves & Rank & Shiptons & Odlum \\
Doves & 6 & 26 & 0 & 6 & 0 \\
Rank & 0 & 0 & 29 & 1 & 1 \\
Shiptons & 5 & 0 & 4 & 25 & 0 \\
Odlum & 0 & 1 & 1 & 0 & 32 \\
\hline $\begin{array}{l}\text { Correct } \\
\text { Proportion }\end{array}$ & 67.6 & 76.5 & 85.3 & 71.4 & 94.1 \\
\hline
\end{tabular}

The factor analysis performed on the variables selected by SDA accounted for $95.9 \%$ of the total variance of the samples (Fig. 1A). The first factor accounted for $66.9 \%$ of the total variance and was highly correlated to $\mathrm{F}_{1}, \mathrm{~F}_{2}, \mathrm{~F}_{5}$ and $\mathrm{F}_{9}$. Consequently, this factor was related to the size of the primitives and the amount of orderliness and uniformity of patches in the image. The second factor accounted for $28.9 \%$ of the total variance and presented high correlation to $\mathrm{F}_{12}$ and $\mathrm{F}_{4}$. Thus, factor 2 was related to the presence of uncorrelated scenes in the image, i.e. as the factor 2 increased, the image heterogeneity (primitives of different greylevel intensities) increased. Fig. 1A shows noticeable segregation between bread crumb grain produced by the organic flours and bread crumb grain produced by the control flour (Odlum). 


\section{$\underline{\text { Neighbourhood grey-level difference matrix method }}$}

The SDA selected the variables coarseness $\left(f_{\text {cos }}\right)$, contrast $\left(f_{\text {con }}\right)$, complexity $\left(f_{\text {com }}\right)$ and strength $\left(f_{\text {str }}\right)$ from the textural features of the neighbourhood grey level difference matrix. These variables were able to correctly identify the treatments in an average of $85.4 \%$ (range 71 97\%). The bread crumb grains produced with Odlum and Doves reached the best classification rates of $97.1 \%$ and $91.2 \%$, respectively (Table 2 ).

The variables $\mathrm{f}_{\text {cos }}$ and $\mathrm{f}_{\text {str }}$ correlated very well with factor 1 which accounted for $57.2 \%$ of the total variance. Hence, as the image texture becomes coarser and the primitives are more easily definable, factor 1 increases. Factor 2 was mainly correlated with $\mathrm{f}_{\text {con }}$ and it comprised $35.3 \%$ of the total variance. Accordingly, as the contrast of the image increased, factor 2 decreased. The contrast of a bread crumb image depends upon the distribution of pore sizes. So, the factorial map in Fig. 1B indicates that Rank presented a coarser texture (higher Factor 1) than Odlum while the latter had a much wider distribution of pore size (higher contrast and hence a lower value of Factor 2) than Doves. In total, the principal components accounted for $92.5 \%$ of the total variance of samples.

Table 2. Classification performance to segregate images by flour type using the method of neighbourhood grey-level difference matrix

\begin{tabular}{lccccc}
\hline \multirow{2}{*}{$\begin{array}{l}\text { Flour } \\
\text { type }\end{array}$} & Allied & Doves & Rank & Shiptons & Odlum \\
\hline Allied & 24 & 2 & 0 & 4 & 0 \\
Doves & 5 & 31 & 0 & 0 & 1 \\
Rank & 0 & 0 & 29 & 2 & 0 \\
Shiptons & 5 & 0 & 4 & 29 & 0 \\
Odlum & 0 & 1 & 1 & 0 & 33 \\
\hline Correct & 70.6 & 91.2 & 85.3 & 82.9 & 97.1 \\
Proportion & & & & & \\
\hline
\end{tabular}

\section{Geometrical method}

In the geometrical method, the textural features that yielded high discriminant capacity among treatments were the maximum number of connected components $\left(\mathrm{NOC}_{\max }\right)$, the average number of connected components $\left(\mathrm{NOC}_{\mathrm{aver}}\right)$, the sample average of number of connected components $\left(\mathrm{NOC}_{\mathrm{sam}}\right)$, the sample standard deviation of the number of connected components $\left(\mathrm{NOC}_{\text {stdev }}\right)$ and the sample standard deviation of the irregularity of the connected components $\left(\right.$ IRGL $\left._{\text {stdev }}\right)$. Together, they rendered an average of correct sample identification of $80.1 \%$ (range $62-97 \%$ in Table 3). The bread crumb grain produced with Odlum and Rank achieved the highest classification rates of $97.1 \%$ and $85.3 \%$, respectively.

The rotated loadings for factor 1 were high in $\mathrm{NOC}_{\max }, \mathrm{NOC}_{\mathrm{aver}}$ and $\mathrm{NOC}_{\mathrm{sam}}$ and explained the $54.6 \%$ of the samples variability. This factor is thus linked to the number of cells of the bread crumb image. Factor 2 had high loadings in $\mathrm{NOC}_{\text {stdev }}$ and $\mathrm{IRGL}_{\text {stdev }}$, and accounted for $36.6 \%$ of the variance. Hence, this factor is related to the variability of cell irregularity (compactness). The factorial map (Fig. 1C) suggests that breads made with Odlum presented high number of cells (higher Factor 1) and a wide range of cell irregularity (higher Factor 2) than Rank. Visually, the gas cells of the bread made with Rank were more compact (more 
rounded) than the ones of the bread made with Odlum. The factor analysis for the textural features from the geometrical method accounted in total for $91.2 \%$ of the total variability.

Table 3. Classification performance to segregate images by flour type using the geometrical method

\begin{tabular}{lccccc}
\hline \multirow{2}{*}{$\begin{array}{l}\text { lour } \\
\text { type }\end{array}$} & \multicolumn{5}{c}{ Classification with Cross-Validation } \\
\cline { 2 - 6 } Allied & 21 & Doves & Rank & Shiptons & Odlum \\
Doves & 6 & 27 & 0 & 5 & 0 \\
Rank & 0 & 0 & 29 & 1 & 1 \\
Shiptons & 7 & 0 & 5 & 27 & 0 \\
Odlum & 0 & 1 & 0 & 0 & 33 \\
\hline $\begin{array}{l}\text { Correct } \\
\text { Proportion }\end{array}$ & 61.8 & 79.4 & 85.3 & 77.1 & 97.1 \\
\hline
\end{tabular}

\section{Method of spectral features insensitive to additive noise}

For Liu and Jernigan's spectral method, the textural featured selected by SDA as best discriminators were isotropy $\left(\mathrm{f}_{7}\right)$, principal component squared $\left(\mathrm{f}_{14}\right)$, energy in the quadrants I and II of the power spectrum $\left(\mathrm{f}_{20}, \mathrm{f}_{21}\right)$ and the relative entropy $\left(\mathrm{f}_{25}\right)$. These five variables were able to correctly identify, in average, $80.1 \%$ of the samples within the treatments (range 64$97 \%$ in Table 4). Odlum and Rank showed the highest classification rates of $97.1 \%$ and $85.3 \%$, respectively.

Table 4. Classification performance to segregate images by flour type using the spectral method insensitive to additive noise

\begin{tabular}{lccccc}
\hline Flour & \multicolumn{5}{c}{ Classification with Cross-Validation } \\
\cline { 2 - 6 } type & Allied & Doves & Rank & Shiptons & Odlum \\
\hline Allied & 22 & 5 & 1 & 2 & 0 \\
Doves & 6 & 27 & 0 & 1 & 1 \\
Rank & 0 & 0 & 29 & 6 & 0 \\
Shiptons & 6 & 1 & 4 & 26 & 0 \\
Odlum & 0 & 1 & 0 & 0 & 33 \\
\hline Correct & 64.7 & 79.4 & 85.3 & 74.3 & 97.1 \\
Proportion & & & & & \\
\hline
\end{tabular}

The variables $f_{20}, f_{21}$ and $f_{14}$ had high loadings in the first factor, which accounted for $52 \%$ of the total variance. As these factors are highly correlated to the energy of the power spectrum, factor 1 was linked to the coarseness of the bread crumb image. The second factor, which accounts for $27.5 \%$ of the total variability, correlated with $f_{7}$ and $f_{25}$. Thus, the factor 2 increases with increasing heterogeneity and decreasing isotropy of bread crumb images (Fig. 1D). The bread crumb grain produced with Rank presented in average higher level of isotropy than the rest of the treatments. This was consistent with the appearance of the bread crumb as its pores were visibly more round-shaped than the other treatments. Therefore, the level of bread crumb isotropy is also related to the elongation of cells. As the cell shape is less elongated and more rounded, the level of isotropy of the image will tend to increase $(\mathrm{P}<0.001$ 
for both regression analyses between isotropy with mean cell eccentricity $(\mathrm{R}=0.43)$ and isotropy with mean cell compactness $(\mathrm{R}=0.42)$. The factorial map (Fig. 1D) shows a higher degree of overlap between treatments especially for Allied and Doves. Besides, the control Odlums presented some degree of overlapping with the organic flour treatments unlike the statistical method maps (Fig. 1A, 1B and 1C). Thus, this method was not as discriminating as the statistical methods.
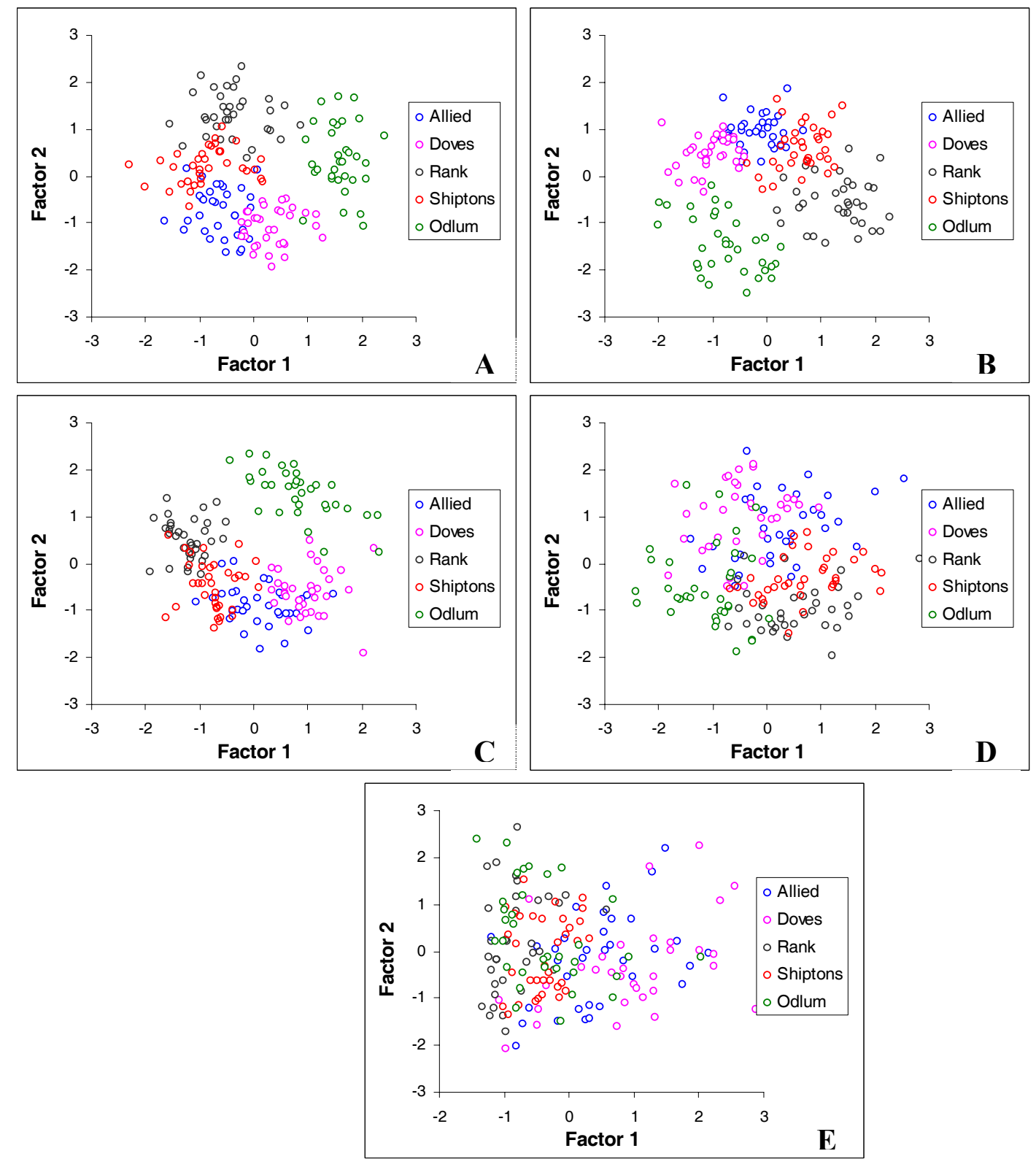

Figure 1. Principal component analysis of bread crumb textural features of slices from five flour types extracted by the following methods: (A) grey-level co-occurrence matrices, (B) neighbourhood grey-level difference matrix, (C) geometrical method, (D) Liu and Jernigan method, and (E) wedges and rings from the Fourier power spectrum 


\section{$\underline{\text { Power spectrum rings and wedges }}$}

From the rings and wedges from the power spectrum, SDA selected the following variables of high discriminant capacity: wedge with centre in $0^{\circ}$ intersected with ring of radius 32 to 64 $\left(\mathrm{W} 0^{\circ} \cap \mathrm{R}_{32-64}\right)$, wedge with centre in $0^{\circ}$ intersected with ring of radius 8 to 16 (W_0 $\left.0^{\circ} \cap \mathrm{R}_{8-16}\right)$, wedge with centre in $135^{\circ}$ intersected with ring of radius 32 to 64 ( $\left.\mathrm{W}_{-} 135^{\circ} \cap \mathrm{R}_{32-64}\right)$, wedge with centre in $135^{\circ}\left(\mathrm{W}_{-} 135^{\circ}\right)$, wedge with centre in $90^{\circ}\left(\mathrm{W}_{-} 90^{\circ}\right)$ and ring of radius 8 to 16 $\left(\mathrm{R}_{8-16}\right)$. These features identified the treatments in 58.5\% on average (range $\left.29-79 \%\right)$ and the discrimination of the control was the poorest of all textural methods $(29.4 \%)$ (Table 5).

The ring and wedges variables accounted only for $75.4 \%$ of the total variance of samples. Factor 1 was correlated to variables containing rings information, i.e. coarseness information (W_0 $0^{\circ} \cap \mathrm{R}_{32-64}, \mathrm{~W}_{-} 0^{\circ} \cap \mathrm{R}_{8-16}, \mathrm{~W}_{-} 135^{\circ} \cap \mathrm{R}_{32-64}$, and $\mathrm{R}_{8-16}$ ) and comprised $52.5 \%$ of the total variance. On the contrary, the second factor was related to the wedges information, i.e. information of image orientation ( $\mathrm{W} \_135^{\circ}$ and $\mathrm{W} \_90^{\circ}$ ) and comprised $22.9 \%$ of the total variance. Fig. 1E shows a very unclear dispersion of the treatments. They were highly overlapped and their regions were not visibly defined.

Table 5. Classification performance to segregate images by flour type using the spectral method of wedges and rings

\begin{tabular}{lccccc}
\hline Flour & \multicolumn{5}{c}{ Classification with Cross-Validation } \\
\cline { 2 - 6 } type & Allied & Doves & Rank & Shiptons & Odlum \\
\hline Allied & 21 & 9 & 0 & 1 & 2 \\
Doves & 7 & 20 & 0 & 1 & 4 \\
Rank & 0 & 0 & 27 & 6 & 8 \\
Shiptons & 2 & 4 & 5 & 22 & 10 \\
Odlum & 4 & 1 & 2 & 5 & 10 \\
\hline Correct & 61.8 & 58.8 & 79.4 & 62.9 & 29.4 \\
Proportion & & & & & \\
\hline
\end{tabular}

In order to determine the textural features with highest discrimination capacity of the bread crumb grain produced with the flours tested, SDA was carried out for a matrix containing all the textural features (64 in total) extracted from the five texture analysis methods. Eleven variables were selected and their interrelationships and underlying structure investigated by factor analysis. A three-principal-components solution was found to be appropriate and accounted for a total variability of $93 \%$. The factors were labelled as crumb coarseness, crumb homogeneity and crumb isotropy.

\section{Conclusions}

Bread crumb grain appearance can be characterised by using digital texture analysis. The textural features from the neighbourhood grey-level difference matrix showed a high discrimination capability as they classified correctly $85 \%$ of the samples using crossvalidation. The geometrical method and the spectral features insensitive to additive noise classified correctly $80 \%$ of the samples. However, higher percentage of correct classification (94 to 97\%) was reached for the control samples (Odlum) using features from the cooccurrence matrices, the neighbourhood difference matrix, the geometrical method and the spectral features insensitive to additive noise. As a whole the statistical texture analysis 
methods depicted the bread crumb grain visual texture better than did the transform methods as the factor analysis of two principal components for the statistical methods explained 91.2 $95.9 \%$ of the total variability of samples. The method of wedges and rings from the Fourier power spectrum performed poorly for distinguishing the bread crumb grains produced with the different flours. Stepwise discriminant analysis carried out over all textural features selected eleven features with the highest discriminant capacity and factor analysis identified the presence of three major components in which the bread crumb grain appearance normally varied. The three-principal-component model accounted for $93 \%$ of the total variability and they were labelled as crumb coarseness, crumb homogeneity and crumb isotropy.

\section{Acknowledgments}

This research was financed by the Irish Department of Agriculture through the Food Institutional Research Measure supported by Irish and EU funds.

\section{References}

- Amadasun, M., and King, R. 1989. Textural features corresponding to textural properties. IEEE Transactions on Systems, Man and Cybernetics, 19, 5, 1264-1274.

- Barrett, A. H., and Peleg, M. 1992. Cell size distributions of puffed corn extrudates. Journal of Food Science, 57, 1, 146-154.

- Bassett, O., Buquet, B., Abouelkaram, S., Delachartre, P., and Culioli, J. 2000. Application of texture image analysis for the classification of bovine meat. Food Chemistry, 69, 437-445.

- Bertrand, D., Le Guernevé, C., Marion, D., Devaux, M., and Robert, P. 1992. Description of the textural appearance of bread crumb by video image analysis. Cereal Chemistry, 69, 257261.

- Chen, Y. Q., Nixon, M., and Thomas, D. W. 1995. Statistical geometrical features for texture classification. Pattern Recognition, 28, 4, 537-552.

- Der, G., and Everitt, B. S. 2002. A Handbook of Statistical Analyses using SAS. Second Edition. Chapman \& Hall. Boca Raton.

- Hair, J. F., Anderson, R., Tatham, R., and Black, W. 1998. Multivariate Data Analysis. Prentice Hall. Fifth Edition. New Jersey. 720 p.

- Haralick, R. M., Shanmugam, K., and Dinstein, I. 1973. Textural features for image classification. IEEE Transactions on Systems, Man, and Cybernetics, 3, 6, 610-621.

- Jain, A. K. 1989. Fundamentals of Digital Image Processing. Prentice Hall. New Jersey. 569 p.

- Keehan, D., Gallagher, E., and Gormley, T. R. 2003. Evaluating the baking potential of organic flours. In "Proceedings of the 12th World Congress of Food Science and Technology", IFT, Chicago, Illinois.

- Li, J., Tan, J., Martz, F. A., and Heymann, H. 1999. Image texture features as indicators of beef tenderness. Meat Science, 53, 17-22.

- Li, J., Tan, J., and Shatadal, P. 2001. Classification of tough and tender beef by image texture analysis. Meat Science, 57, 341-346.

- Liu, S.S., and Jernigan, M.E. 1990. Texture analysis and discrimination in additive noise. Computer Vision, Graphics, and Image Processing, 49, 52 - 67.

- Majumdar, S. and Jayas, D. S. 2000. Classification of cereal grains using machine vision: III. Texture models. Transactions of the ASAE, 43, 1681-1687.

- McCauley, J. D., Thane, B. R., and Whittaker, A. D. 1994. Fat estimation in beef ultrasound images using texture and adaptive logic networks. Transactions of the ASAE, 37, 997-1002. 
- Park, B., and Chen, Y. R. 1996. Multispectral image co-occurrence matrix analysis for poultry carcasses inspection. 97-110 p. In "Food Processing Automation III - Proceedings of the FPAC Conference". St. Joseph, ASAE Michigan.

- Shiranita, K., Miyahima, T., and Takiyama, R. 1998. Determination of meat quality by texture analysis. Pattern Recognition Letters, 19, 1319-1324.

- Weszka, J.S., Dyer, C. R., and Rosenfeld, A. 1976. A comparative study of texture measures for terrain classification. IEEE Transactions on Systems, Man, and Cybernetics, 6, 4, 269 285.

- Whittaker, A. D., Park, B., Thane, B. R., Miller, R. K., and Savell, J. W. 1992. Principles of ultrasound and measurement of intramuscular fat. Journal of Animal Science, 70, 942-952.

- Zayas, I. Y. 1993. Digital image texture analysis for bread crumb grain evaluation. Cereal Food World, 38, 10, 760 - 766. 